Modern Physics Letters A

(c) World Scientific Publishing Company

\title{
CHIRAL SYMMETRY IN NUCLEI THEORETICAL EXPECTATIONS AND HARD FACTS
}

\author{
ULRICH MOSEL \\ Institut für Theoretische Physik, Universitaet Giessen \\ Giessen, D-35392 , Germany* \\ Received (Day Month Year) \\ Revised (Day Month Year)
}

\begin{abstract}
It is widely believed that chiral symmetry is restored not only at high temperatures, but also at high nuclear densities. The drop of the order parameter of the chiral phase transition, the chiral condensate, with density has indeed been calculated in various models and is as such a rather robust result. In this talk I point out that the connection of this property with actual observables is far less clear. For this task a good hadronic description of the primary production of hadrons, their propagation inside the nuclear medium, their decay and the propagation of the decay products through the medium to the detector all have to be treated with equal accuracy and weight. In this talk I illustrate with the examples of $\omega$ production and $\pi^{0} \pi^{0}$ production how important in particular final state interactions are.

Keywords: hadrons; in-medium properties

21.65.Jk, 25.20.-x, 13.60.Le
\end{abstract}

\section{Introduction}

The interest in in-medium properties of hadrons has been growing over the last decade because of a possible connection with broken symmetries of QCD and their partial restoration inside nuclear matter. Nearly two decades ago there were sophisticated theoretical predictions that masses of vector mesons should generally decrease in medium as a function of density due to a partial restoration of chiral symmetry 211. Specifically, there existed well-worked out predictions that the scalar $2 \pi$ strength should decrease in medium and that the vector meson masses should drop 413. All of these calculations were performed for idealized situations (infinite cold nuclear matter at rest) and little attention was paid to the actual observability of these predicted changes. At the same time experiments (CERES, TAPS) seemed to show the predicted behavior. The CERES results indicated a significant broadening of the $\rho$ meson in medium 5 , whereas the TAPS results on the $2 \pi$ strength exhibited the predicted lowering of the $\sigma$ strength inside nuclei $[6$. Most recently, the 
CB/TAPS@ELSA experiment has also obtained an indication for a lowering of the $\omega$ meson mass in nuclei ${ }^{7}$. Other interesting data in this context have been obtained by groups at JLAB $\frac{8}{8}, \mathrm{KEK}^{-9}$ and CERN 10 . While experiments with relativistic or ultrarelativistic heavy ions have the advantage that high densities can be produced, they have the disadvantage that any observed signal naturally is a time-integral over the whole collision history. On the other hand, experiments with microscopic probes on cold nuclei have the advantage that they proceed closer to equilibrium, even though the densities probed are much lower.

We have focussed our work on in-medium properties of hadrons on two steps. First, we have performed state-of-the-art calculations of vector meson spectral functions in cold nuclear matter. Second, we have followed closely the CB/TAPS@ELSA experiment and have performed various feasibility studies and analyses of this experiment searching for in-medium changes of the $\omega$ meson in medium. In a third step we have also analyzed the lowering of scalar strength in nuclei, predicted as a signature for chiral symmetry restoration, and indeed observed by the TAPS experiment.

\section{In-Medium Properties of Vector Mesons}

\subsection{QCD sum rules and hadronic models}

While the predicted drop of the chiral condensate with density 11 is a rather robust result the connection between chiral condensates on one hand and hadronic spectral functions on the other cannot simply be inferred from looking at the dependence of the condensate on density and temperature. Instead, the connection is much more indirect; only an integral over the spectral function can be linked via the QCD sum rule to the condensate behavior in medium. In an abbreviated form this connection is given by

$$
R^{\mathrm{OPE}}\left(Q^{2}\right)=\frac{\tilde{c}_{1}}{Q^{2}}+\tilde{c}_{2}-\frac{Q^{2}}{\pi} \int_{0}^{\infty} d s \frac{\operatorname{Im} R^{\mathrm{HAD}}(s)}{\left(s+Q^{2}\right) s}
$$

with $Q^{2}:=-q^{2} \gg 0$ and some subtraction constants $\tilde{c}_{i}$. Here $R^{\mathrm{OPE}}$ represents a Wilson's operator expansion of the current-current correlator in terms of quark and gluon degrees of freedom in the space-like region. It is an expansion in terms of powers of $1 / Q^{2}$ and contains the condensates as expansion parameters. On the other hand, $R^{\mathrm{HAD}}(s)$ in (1) is the same object for time-like momenta, represented by a parametrization in terms of hadronic variables. The dispersion integral connects time- and space-like momenta. Eq. (1) connects the hadronic with the quark world. It allows to determine parameters in a hadronic parametrization of $R^{\mathrm{HAD}}(s)$ by comparing the lhs of this equation with its rhs. Invoking vector meson dominance it is easy to see that for vector mesons $\operatorname{Im} R^{\mathrm{HAD}}(s)$ in (1) is just the spectral function of the meson under study.

The operator product expansion of $R^{\mathrm{OPE}}$ on the lhs involves quark- and gluon 
condensates 1213; ; of these only the two-quark condensates are reasonably well known, whereas our knowledge about already the four-quark condensates is rather sketchy.

Using the measured, known vacuum spectral function for $R^{\mathrm{HAD}}$ allows one to obtain information about these condensates appearing on the lhs of (11). On the other hand, modelling the density-dependence of the condensates yields information on the change of the hadronic spectral function when the hadron is embedded in nuclear matter. Since the spectral function appears under an integral the information obtained is not direct. Therefore, as Leupold et al. have shown 12113, the QCDSR provides important constraints for the hadronic spectral functions in medium, but it does not fix them. Kaempfer et al have turned this argument around by pointing out that measuring an in-medium spectral function of the $\omega$ meson could help to determine the density dependence of the higher-order condensates 14 . Leupold has also argued that the four-quark condensates are interesting, because a special one of them can be unambiguously linked to a difference of spectral functions in the axial and vector channel 15 .

Thus models are needed for the hadronic interactions. The quantitatively reliable ones can at present be based only on 'classical' hadrons and their interactions. In lowest order in the density the mass and width of an interacting hadron in nuclear matter at zero temperature and vector density $\rho_{v}$ are given by (for a meson, for example)

$$
\begin{aligned}
m^{* 2} & =m^{2}-4 \pi \mathcal{R} e f_{m N}\left(q_{0}, \theta=0\right) \rho_{v} \\
m^{*} \Gamma^{*} & =m \Gamma^{0}-4 \pi \mathcal{I} m f_{m N}\left(q_{0}, \theta=0\right) \rho_{v} .
\end{aligned}
$$

Here $f_{m N}\left(q_{0}, \theta=0\right)$ is the forward scattering amplitude for a meson with energy $q_{0}$ on a nucleon. The width $\Gamma^{0}$ denotes the free decay width of the particle. For the imaginary part this is nothing other than the classical relation $\Gamma^{*}-\Gamma^{0}=v \sigma \rho_{v}$ for the collision width, where $\sigma$ is the total cross section. This can easily be seen by using the optical theorem. The calculation of hadronic spectral functions in medium thus reduces - in lowest order in the density - to a calculation of hadron-nucleon scattering amplitudes.

Unfortunately it is not a-priori known up to which densities the low-density expansion (2) is useful. Post et al. 16 have recently investigated this question in a coupled-channel calculation of selfenergies. Their analysis comprises pions, $\eta$ mesons and $\rho$-mesons as well as all baryon resonances with a sizeable coupling to any of these mesons. The authors of $\frac{16}{16}$ find that already for densities less than $0.5 \rho_{0}$ the linear scaling of the selfenergies inherent in (2) is badly violated for the $\rho$ and the $\pi$ mesons, whereas it is a reasonable approximation for the $\eta$ meson. This may serve as a warning sign for many in-medium calculations that use the lowdensity approximation without any further proof of its reliability. Reasons for this deviation from linearity are Fermi-motion, Pauli-blocking, selfconsistency and shortrange correlations. For different mesons different sources of the discrepancy prevail: 
for the $\rho$ and $\eta$ mesons the selfconsistency acts against the low-density theorem by inducing a strong broadening for the $D_{13}(1520)$ and a slightly repulsive mass shift for the $S_{11}(1535)$ nucleon resonances to which the $\rho$ and the $\eta$ meson, respectively, couple. The investigation of in-medium properties of mesons, for example, thus involves at the same time the study of in-medium properties of nucleon resonances and is therefore a coupled-channel problem.
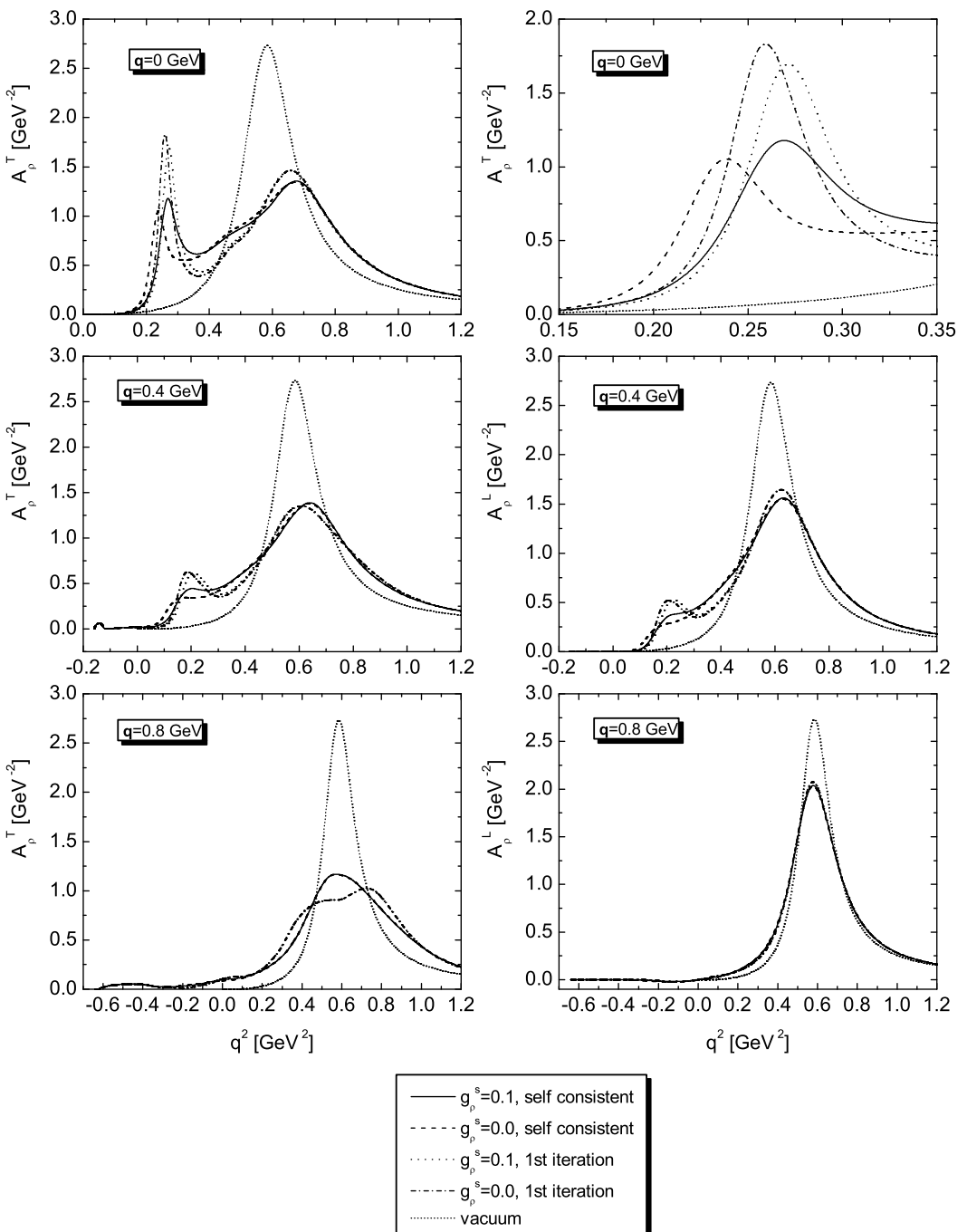

Fig. 1. Spectral function of the $\rho$ meson in nuclear matter at density $\rho_{0}$ for various momenta indicated in the figure. The left column shows the transverse spectral function, the right column that of longitudinally polarized $\rho$ mesons. The thin dotted line in each figure is the vacuum spectral function, the other curves give the effect of selfconsistency and short-range correlations (from 16 ). 
The strong interplay between the $\rho$ meson and the D13(1520)-nucleon hole excitation leads to a dominant lower hump in the $\rho$ spectral function also in this relativistic and selfconsistent calculation; it confirms our earlier result obtained in a more simplified approach. Whereas the longitudinal component of the $\rho$ meson only broadens somewhat, the transverse component shows a major distortion which evolves as a function of the $\rho$ momentum (see Fig. 1) and reflects the coupling to the D13(1520) resonance. It is obvious that such a rich structure cannot be obtained from QCD sumrules that involve only an integral over the spectral function. At the same time, the D13(1520) resonance broadens considerably due to the opening of phase-space for $\rho$-decay. For the $\eta$ meson the optical potential resulting from our model is rather attractive whereas the in-medium modifications of the S11(1535) are found to be quite small.

\subsection{Coupled channel calculation of hadronic selfenergies}

Besides the range of validity of the low-density approximation the meson-nucleon cross sections are often not directly measurable and can be inferred only from unitarity arguments involving many coupled channels. Unitarity thus provides important contraints for reactions such as $\omega N \rightarrow 2 \pi N$ that are experimentally not accessible, but are not only needed for but contribute significantly to a calculation of the $\omega$ selfenergy. Bearing this in mind we have recently performed a calculation of the selfenergy of the $\omega$ meson in medium. This calculation is based on a unitary cou-

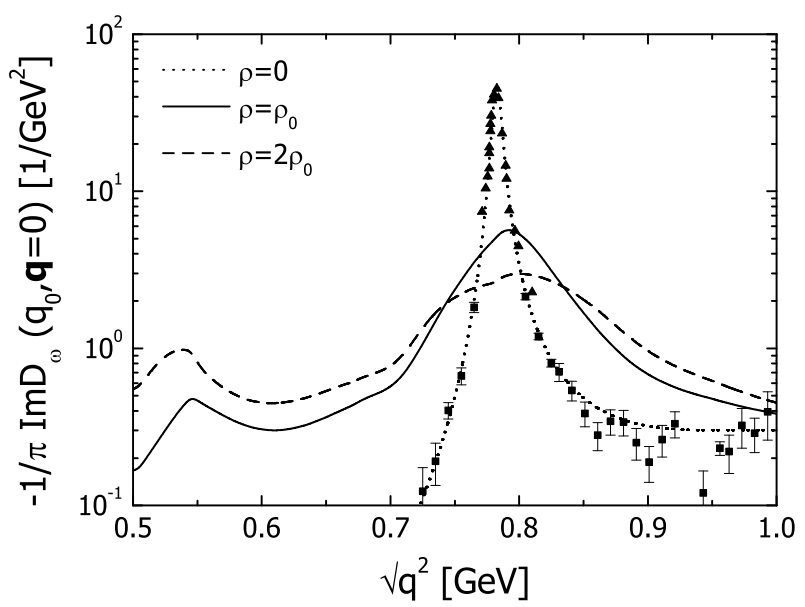

Fig. 2. Spectral function of the $\omega$ meson in nuclear matter at rest, at densities $0, \rho_{0}$ and $2 \rho_{0}$ (from 17 .

pled channel analysis of all existing $\pi N$ and $\gamma N$ data up to an invariant mass of $2 \mathrm{GeV}$ 17. The coupled channel character of this calculation is of utmost importance here because it is the only way to include experimental constraints on the 
$2 \pi$ decay channel that was found to be dominant in 3 . This analysis and thus also the selfenergy of the $\omega$ meson extracted from the $\omega N$ scattering amplitude gives a broadening of about $60 \mathrm{MeV}$ at $\rho_{0}$ for an $\omega$ at rest and a small upward shift of the peak mass. In addition, due to a nonzero coupling of the $\omega$ to the S11(1535) resonance the $\omega$ spectral function exhibits a small peak at a mass of around $550 \mathrm{MeV}$. This calculation gives, for the first time, the $\omega$ selfenergy also for nonzero momenta (which corresponds to the experimental situation). The result of this calculation is shown in Fig. 2,

For vanishing $\omega$ momentum this result qualitatively agrees with that of 18 in that it yields a lower mass structure in the spectral function at an invariant mass of $500-600 \mathrm{MeV}$ and only a very small shift of the main peak; the latter is in contrast to the results of ${ }^{3}$ (for a recent discussion of the results obtained in ${ }^{3}$ see 19 ) and of 20. The latter was based on a relativistic mean-field model and does not contain any dispersive effects. Fig. 2 also shows clearly that the $\omega$ meson in medium acquires a significant broadening, caused by collisions with the surrounding nucleons.

\subsection{Data analysis with GiBUU}

Our second line of approach to the problem of in-medium selfenergies has concentrated on an analysis of the recent CB/TAPS@ELSA data ${ }^{7}$ Since the experiment looks for the channel $\gamma+A \rightarrow A^{*}+\omega \rightarrow A^{*}+\gamma+\pi^{0}$ it is mandatory to control the effects of final state interactions on the $\pi^{0}$ in a quantitative way. The only method available for this is that of coupled channel semiclassical transport calculations which - as we had shown earlier in extensive work - can give a consistent description of many experimental phenomena, both in heavy-ion 22 as well as in nucleon-, pion- 23 , photon- $\frac{24}{}$ and neutrino-induced reactions 25 . For any reaction on nuclei with hadrons in the final state a state-of-the-art transport calculation of the final state interactions is an indispensible part of the theory. We have, therefore, spent significant effort on developing a new code, dubbed 'GiBUU', for the transport calculations. This code is written in object-oriented FORTRAN 95/2003 and incorporates all the experience we have gained with earlier numerical implementations at Giessen over the last 20 years 21 . A major effort has gone into an analysis of the $\omega$ photo-production experiment at CB/TAPS@ELSA.

For $\omega$ mesons the CB/TAPS@ELSA collaboration has measured the nuclear transparency 26127, see Fig. 3. This transparency gives directly the imaginary part of the meson's selfenergy in medium; using a low-density approximation one can then extract the inelastic $\omega N$ cross section. The figure shows an analysis of these data for various values of the collisional width, assumed at a momentum of $1.1 \mathrm{GeV}$, the average momentum of the $\omega$ mesons in this experiment. Again, care has been taken to ensure consistency between the collisional width and the inelastic $\omega N$ cross sections. It is seen from this figure that a width of about $130-150 \mathrm{MeV}$ describes the data. This width is larger by about a factor of 3 than that previously assumed. A fit to the data can actually also determine the momentum-dependence of this cross 
section 27. With this method we have also analysed results on the experimental

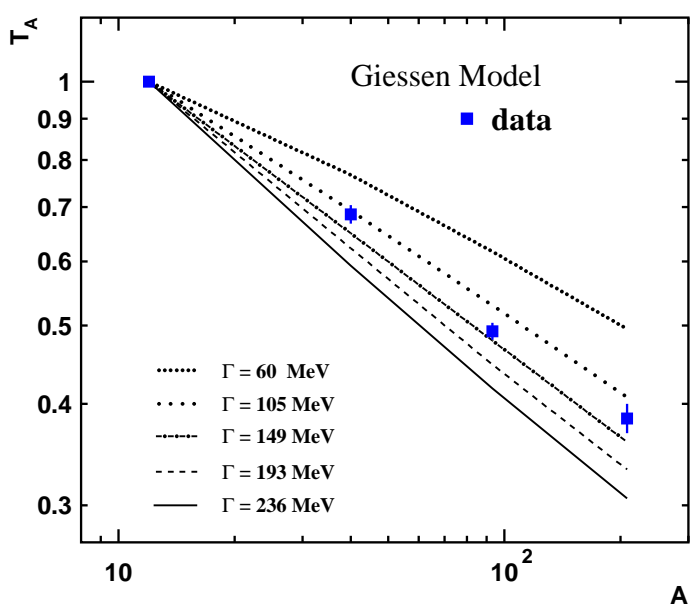

Fig. 3. Experimentally determined transparency ratio (squares) as a function of massnumber in comparison with GiBUU calculations assuming various values for the inleastic width at an $\omega$ momentum of $1.1 \mathrm{GeV}$ (from 26|27).

determination of the nuclear transparency ratio for $\phi$ mesons 28 , measured by a group at SPRING8. Also in this case an unexpectedly large inelastic cross section for $\phi N$ interactions was extracted. We have found that indeed cross sections about a factor 3 larger than those theoretically expected are needed to explain the mentioned data, in line with a simple Glauber analysis by the SPRING8 group.

Our simulations give a full event analysis and thus allow to calculate also background contributions on the same footing as the actual signal. They also allow insight into the effects of rescattering of the pions produced in the decay of the $\omega$ meson and have suggested a method to suppress the rescattered pion background that has actually been adopted by the experimental group. The result of this analysis is that the $\omega$ spectral function published by the CB/TAPS@ELSA collaboration can be explained only if a lowering of the $\omega$ meson mass in medium by about $16 \%$ is assumed together with the appropriate collisional broadening.

A problem in this context is that the experiment does not determine the spectral function of the $\omega$ meson itself. Instead, we have noted that the result of the experimental analysis is the product of the primary production cross section with the spectral function and the partial decay probability into the channel under study $\left(\pi^{0} \gamma\right.$ here). If the first and the latter depend strongly on the invariant mass itself, 
as it does for the CB/TAPS@ELSA experiment, then significant distortions of the spectral function may arise. This is a topic that is presently under intensive study 29 .

\section{In-medium Properties of Scalar Mesons}

Since the restoration of chiral symmetry should lead to a convergence of scalarisoscalar and scalar-isovector strength at high densities it was predicted that the decay of the scalar $\sigma$ meson into two uncharged pions should become increasingly difficult and the scalar strength should become narrower at the $2 \pi$ threshold 4 . The TAPS collaboration had indeed initially seen this effect in the $2 \pi^{0}$ data as predicted, whereas a comparison measurement in the $\pi^{0} \pi^{ \pm}$channel did not show such an effect 6 . Various explanations for these findings have been advanced by the Valencia group 30 and by a group in Lyon 31 in terms of $\pi-\pi$ correlations or chiral symmetry restoration in nuclei.

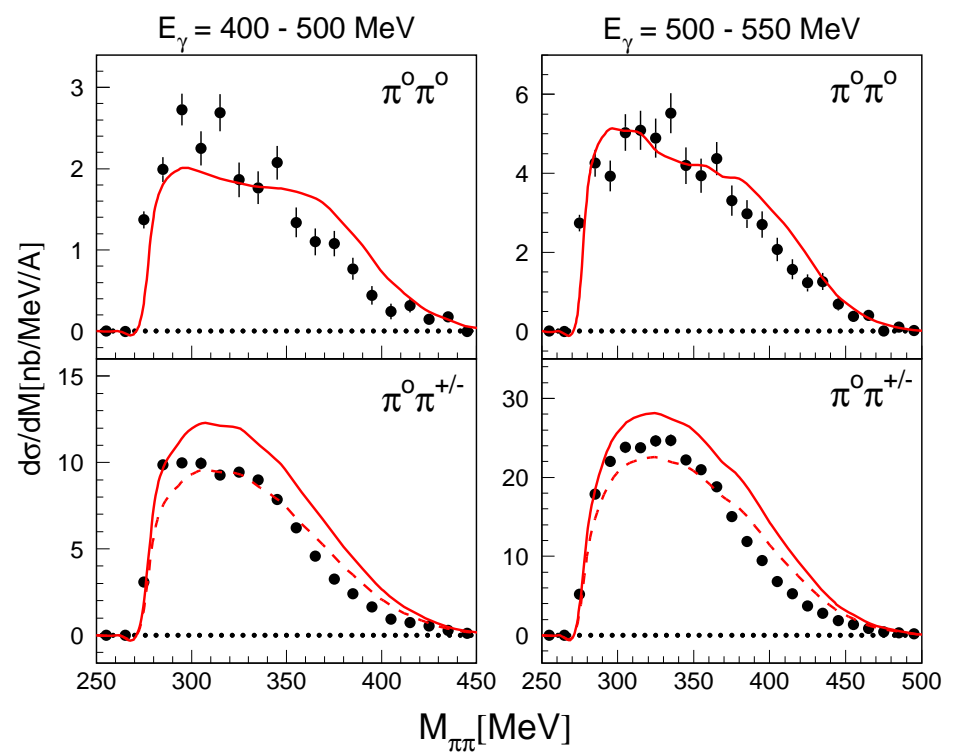

Fig. 4. Data of the TAPS collaboration (Bloch et al.) for $2 \pi^{0}$ photoproduction on a ${ }^{40} \mathrm{Ca}$ target for two different photon energies. The solid curve gives the result of a GiBUU calculation [32, the dashed curves in the semicharged $2 \pi$ channel are normalized to the data (see text). Data from 33 .

None of these calculations, however, did look into the simplest possible explanation of the observed effects in terms of mundane pion rescattering. We have, therefore, performed such calculations 23 using the GiBUU method which is ideally suited for this task. These calculations, which did not contain any effects connected with $\pi \pi$ correlations, did reproduce the observed effect for the $\pi^{0} \pi^{0}$ channel, but 
they also predicted a similar effect in the semi-charged channel where it had not been seen experimentally. However, a more recent analysis with higher statistics by Bloch et al. 33 yielded a result for both charge channels that is in perfect agreement with our calculations (see Fig. 4 the dashed lines in this figure are normalized in height to the data, this normalization reflects uncertainties in the elementary cross sections). In particular the yield in the semi-charged channel is strongly influenced by a coupled-channel effect, the charge transfer in $\pi N$ interactions; Glauber based absorption models miss this contribution. This illustrates that a very sophisticated treatment of final state interactions is absolutely mandatory when looking for more 'exotic' effects in nuclei. We conclude that any analysis of the $2 \pi^{0}$ data with respect to a lowering of the scalar strength in nuclei has to take the pion rescattering effects into account. Present day's data are all consistent with simple rescattering.

\section{Summary}

The main message we have learned from the studies reported here is that it is important to calculate not only in-medium properties under idealized conditions (static, uniform matter in equilibrium), but to also explore the influence of these properties on actual observables. The spectral function itself, which contains the information on in-medium selfenergies, in particular in-medium masses and widths, is not directly observable. Instead, both the creation of the studied hadron as well as its decay influence the observables as much as the spectral function itself and thus have to be under good control. The same holds for the final state interactions on hadronic decay products. Here a state-of-the-art treatment of final state interactions is mandatory. There is now general agreement on the amount of collisional broadening of vector mesons in medium, but the verification of an actual mass-shift still requires more work, both theoretical and experimental. Until then the quest for chiral symmetry restoration in nuclei remains a challenging topic.

\section{Acknowledgement}

This talk is mainly based on results obtained by Oliver Buss and Pascal Muehlich as part of their doctoral theses. I thank them, and the entire GiBUU group, for many inspiring discussions.

\section{References}

1. T. Hatsuda, S. H. Lee and H. Shiomi, Phys. Rev. C 52, 3364 (1995) arXiv:nucl-th/9505005.

2. G. E. Brown and M. Rho, Phys. Rev. Lett. 66 (1991) 2720.

3. F. Klingl and W. Weise, Nucl. Phys. A 606 (1996) 329; F. Klingl, N. Kaiser and W. Weise, Nucl. Phys. A 624 (1997) 527 arXiv:hep-ph/9704398.

4. K. Yokokawa, T. Hatsuda, A. Hayashigaki and T. Kunihiro, Phys. Rev. C 66 (2002) 022201 arXiv:hep-ph/0204163.

5. G. Agakishiev et al. [CERES Collaboration], Phys. Rev. Lett. 75 (1995) 1272. 
6. J. G. Messchendorp et al., Phys. Rev. Lett. 89, 222302 (2002) arXiv:nucl-ex/0205009.

7. D. Trnka et al. [CB/TAPS@ELSA Collaboration], Phys. Rev. Lett. 94, 192303 (2005) arXiv:nucl-ex/0504010.

8. R. Nasseripour, M. H. Wood, C. Djalali, D. P. Weygand, C. Tur, U. Mosel, P. Muehlich, CLAS Collaboration, Phys. Rev. Lett. (2007) in press [arxiv.org/abs/0707.2324v3].

9. S. Yokkaichi et al. [KEK-PS325 Collaboration], Int. J. Mod. Phys. A 22, 397 (2007).

10. S. Damjanovic et al. [NA60 Collaboration], Nucl. Phys. A 783 (2007) 327 arXiv:nucl-ex/0701015.

11. S. Klimt, M. Lutz and W. Weise, Phys. Lett. B 249 (1990) 386.

12. S. Leupold, Phys. Rev. C64, 015202 (2001).

13. S. Leupold, U. Mosel, Phys. Rev. C58, 2939 (1998); S. Leupold et al., Nucl. Phys. A 628, 311 (1998).

14. S. Zschocke, O. P. Pavlenko and B. Kampfer, Phys. Lett. B 562 (2003) 57

15. S. Leupold, AIP Conf. Proc. 842, 168 (2006).

16. M. Post, S. Leupold and U. Mosel, Nucl. Phys. A 741, 81 (2004) arXiv:nucl-th/0309085.

17. P. Muehlich, V. Shklyar, S. Leupold, U. Mosel and M. Post, Nucl. Phys. A 780, 187 (2006) arXiv:nucl-th/0607061.

18. M. F. M. Lutz, G. Wolf and B. Friman, Nucl. Phys. A 706 (2002) 431 [Erratum-ibid. A 765 (2006) 431] arXiv:nucl-th/0112052.

19. F. Eichstaedt, S. Leupold, U. Mosel and P. Muehlich, Prog. Theor. Phys. Suppl. 168, 495 (2007) arXiv:0704.0154 [nucl-th]].

20. K. Saito, K. Tsushima, D. H. Lu and A. W. Thomas, Phys. Rev. C 59, 1203 (1999) arXiv:nucl-th/9807028.

21. for details see: http://gibuu.physik.uni-giessen.de/GiBUU/

22. A. B. Larionov, O. Buss, K. Gallmeister and U. Mosel, Phys. Rev. C 76 (2007) 044909 arXiv:0704.1785 [nucl-th]].

23. O. Buss, L. Alvarez-Ruso, A. B. Larionov and U. Mosel, Phys. Rev. C 74, 044610 (2006) arXiv:nucl-th/0607016.

24. O. Buss, T. Leitner, U. Mosel and L. Alvarez-Ruso, Phys. Rev. C 76, 035502 (2007) arXiv:0707.0232 [nucl-th]].

25. T. Leitner, L. Alvarez-Ruso and U. Mosel, Phys. Rev. C 73, 065502 (2006) arXiv:nucl-th/0601103.

T. Leitner, L. Alvarez-Ruso and U. Mosel, Phys. Rev. C 74, 065502 (2006) arXiv:nucl-th/0606058.

26. V. Metag, Erice 2007 Proc., arXiv:0711.4709 [nucl-ex].

27. M. Kotulla et al., to be published

28. P. Muhlich and U. Mosel, Nucl. Phys. A 765, 188 (2006) arXiv:nucl-th/0510078.

29. K. Gallmeister, M. Kaskulov and U. Mosel, Erice 2007 Proc., arXiv:0712.2200 [nuclth].

30. L. Roca, E. Oset and M. J. Vicente Vacas, Phys. Lett. B 541, 77 (2002) arXiv:nucl-th/0201054.

31. G. Chanfray, Z. Aouissat, P. Schuck and W. Noerenberg, Phys. Lett. B 256, 325 (1991).

32. O. Buss, L. Alvarez-Ruso, P. Muhlich and U. Mosel, Eur. Phys. J. A 29 (2006) 189 arXiv:nucl-th/0603003.

33. F. Bloch et al., Eur. Phys. J. A 32 (2007) 219 arXiv:nucl-ex/0703037. 